Article

\title{
Evaluation of the Food Contact Suitability of Aged Bio-Nanocomposite Materials Dedicated to Food Packaging Applications
}

\author{
Anaïs Lajarrige *(D), Nathalie Gontard, Sébastien Gaucel ${ }^{\mathbb{D}}$ and Stéphane Peyron \\ JRU IATE 1208, CIRAD, INRAE, Montpellier SupAgro, University of Montpellier, 2 place Pierre Viala, \\ F-34060 Montpellier 01, France; nathalie.gontard@inra.fr (N.G.); sebastien.gaucel@inra.fr (S.G.); \\ stephane.peyron@umontpellier.fr (S.P.) \\ * Correspondence: lajarrige.anais@hotmail.fr
}

Received: 8 January 2020; Accepted: 22 January 2020; Published: 28 January 2020

check for updates

\begin{abstract}
Nanocomposite materials based on bio-polyesters (PBSA and PHBV) have been evaluated for their suitability for food contact according to the recommendations defined for non-biodegradable plastic materials, and subsequently, according to accelerated aging treatment. On the basis of the limited number of material/migrant/food simulant combinations studied here, the test for migration, using food simulants, appeared directly applicable to testing such materials which are not considered humidity-sensitive materials. Considering the only compliance criterion that must be met by the materials in contact, the materials submitted to the aging processing are not of safety concern and the incorporation of nanoclays in aged biodegradable materials does not interfere with their inertial properties in a dramatic way. At the molecular scale, the UV irradiation proved to induce an increase in the degree of crystallinity, resulting in a modification of transport properties of both packaging materials. The values of overall migration and specific migration were reduced without decreasing the diffusion coefficients of the target additives. The UV treatment and the addition of nanoparticles, therefore, seem to jointly promote the retention of organic compounds in the materials by increasing their affinity for packaging material.
\end{abstract}

Keywords: nanoclays; biodegradable polymer; accelerated aging; migration; apparent diffusion coefficient $\left(D_{\text {app }}\right)$

\section{Introduction}

In the face of growing environmental concerns, new alternative materials must be proposed, especially for short-term applications like food packaging. In this context, biodegradable polymers made with bio-sourced resources are considered as promising materials. Among the biodegradable polymers, aliphatic polyesters such as poly(butylene succinate-co-butylene adipate) (PBSA) and poly(hydroxybutyrate-co-hydroxyvalerate) (PHBV) appear as interesting candidates to replace conventional non-degradable polymers. In addition to their biodegradability, these two polymers have the advantage of being fully (PHBV) or partially (PBSA) bio-sourced. However, to make them competitive towards non-degradable petroleum-based polymers, particularly in terms of barrier properties, their blend with reinforcing nanofillers seems to be a promising alternative widely described in the literature, but has been placed on the market exclusively in synthetic matrices until now.

The introduction of nanofillers in materials intended to be in contact with food raises new questions in terms of consumer safety. The migration of nanoclays from nanocomposite material into food or food simulant has been already studied [1-4]. They concluded that their migration will take place in the case of very small nanoparticles with radii in the order of $1 \mathrm{~nm}$, from polymer matrices 
that have a relatively low dynamic viscosity, and that do not interact with the nanoparticles $[5,6]$. Recently, investigations were performed in the same way on nanocomposites materials exposed to thermal, chemical and mechanical stress followed by mechanical abrasion of their surface. The authors concluded that even under dynamic stress conditions, nano-laponites, considered as exhibiting a high potential to be released from a polymer matrix, do not contaminate the food under a nano-form [7].

In addition to the risks related to the release of nanoparticles into food, the impact of the addition of nanoclays in polymer matrix on the migration of non-nano substances is much less studied. The few studies carried out on conventional plastic materials concluded that the incorporation of nanoclays positively affected the diffusion of migrating substances [8-10]. In the case of biodegradable plastic materials, this is not always verifiable, depending on the nature of the migrating substance, the polymer type and the nature of the food simulant in contact [11].

Biodegradable polymers are, by definition, unstable over time and subject to degradation. These polymer degradations can be classified according to the nature of the causative agents: photo-oxidative degradation, thermal degradation, ozone-induced degradation, mechanochemical degradation, catalytic degradation, and biodegradation [12]. These degradations lead to a modification of their structure and consequently to their properties [13]. Most of the time, the degradation affects the thermal stability, color, mechanical properties, crystallinity and lamellar thickness distribution [14]. These changes may be desirable, as for the end-of-life of the polymer, or undesirable, such as changes during its use in contact with food [15].

From a food safety standpoint, as with any materials intended to contact food, bio-nanocomposite materials are subject to the European framework Regulation (EC) 1935/2004. In the absence of specific regulations dedicated to nanocomposites and bio-nanocomposites, food contact suitability is assessed following the recommendations for non-degradable plastic materials specified in the Regulation (EU) 10/2011. However, in the case of biodegradable nanocomposite materials, for which the structure evolves over time, their food contact ability has to be investigated throughout their use in food contact. In practice, their compliance can be validated after the production of the packaging but can evolve during an extended storage period under detrimental conditions of relative humidity associated with uncontrolled exposure to light. That is why it is important to determine the impact of the polymer degradations on the properties of transfer and inertia of materials.

In this context, this work aims to evaluate the food contact suitability of two distinct biodegradable polyesters (PBSA and PHBV) after accelerated aging (UV, humidity and temperature) and to determine if the recommendations established for non-degradable materials can be applicable to the particular case of biodegradable materials. Neat and nanocomposite materials reinforced with $5 \mathrm{wt} \%$ of organo-modified montmorillonite were considered to determine the impact of the reinforcing nanofillers on the aging of materials, and consequently on their properties of transfer and inertia. In this way, overall and specific migrations were assessed according to the conditions of tests recommended for plastic materials. A panel of additives representative of possible substances added in the polymer formulation were studied and their diffusivities in the polymer matrix were also.

\section{Materials and Methods}

\subsection{Materials}

Poly(butylene succinate-co-butylene adipate) (PBSA, PBE 001, density $1.24 \mathrm{~g} . \mathrm{cm}^{-3}$ ) and poly(hydroxybutyrate-co-hydroxyvalerate) (PHBV, PHI 002, density $1.23 \mathrm{~g} . \mathrm{cm}^{-3}$ ) pellets were commercially procured from Natureplast, France.

Cloisite 30B (C-30B), an organo-modified montmorillonite clay containing a methyl bis-2-hydroxyethyl ammonium quaternary salt with a cation exchange capacity (CEC) of 90 meq/100 g, were supplied by BYK additives and instruments, Wesel, Germany.

An array of eight low molar mass molecules with a variety of chemical and physical properties as described in FDA and EFSA regulations [16,17] was selected for representative 
plastic additives. The additives chosen included: chlorobenzene, methyl salicylate, biphenyl, phenyl cyclohexane, benzophenone, methyl stearate and DEHA, all provided from Sigma-Aldrich, Saint-Quentin-Fallavier, France.

Acetic acid, ethanol and 2,2,4 trimethyl pentane (isooctane) were commercially provided by Sigma-Aldrich with a purity $>99 \%$ and were used to prepare the food simulants for migration experiments: $3 \%$ acetic acid, $95 \%$ ethanol and isooctane.

\subsection{Preparation of Materials}

PBSA and PHBV films containing $5 \mathrm{wt} \%$ C-30B (PBSA NCP and PHBV NCP) were synthetized by a melt extrusion process using an EuroLab 16XL co-rotating twin-screw extruder (Thermo Scientific ${ }^{\mathrm{TM}}$, Karlsruhe Germany) with a L/D ratio of 40 and a screw diameter of $16 \mathrm{~mm}$ as described previously [11]. The control samples of neat polymers were prepared following the same protocol used for nanocomposites in order to respect the same thermal processes.

The neat and nanocomposite films were then subjected to accelerated aging in a Q-SUN Xe-3 xenon arc chamber (Q-LAB, Bolton, England) equipped with Window $Q$ filter. The conditions were as follows: $35^{\circ} \mathrm{C}, 50 \% \mathrm{RH}$ and an irradiance of $1.44 \mathrm{~W} \cdot \mathrm{m}^{-2}$ at $420 \mathrm{~nm}$ for 30 days.

\subsection{Differential Scanning Calorimetry (DSC)}

The crystallinity of each film was studied using a differential scanning calorimeter (DSC series Q200, TA Instruments, New Castle, DE, USA). Experiments were carried out on neat and nanocomposite films, before and after 30 days of accelerated aging. All measurements were performed under a nitrogen atmosphere $\left(50 \mathrm{~mL} \cdot \mathrm{min}^{-1}\right)$. Specimens of 5-8 $\mathrm{mg}$, weighed using a microbalance (Sartorius micro PRO 11), were placed in aluminum sample pans (Tzero Aluminium Hermetic pan, TA Instruments). The thermal protocol was defined according to the polymer. The PBSA samples were heated up to $150{ }^{\circ} \mathrm{C}$, maintained at $150{ }^{\circ} \mathrm{C}$ for $5 \mathrm{~min}$, cooled to $-35^{\circ} \mathrm{C}$ and finally reheated to $150{ }^{\circ} \mathrm{C}$. In the case of PHBV, samples were heated up to $200{ }^{\circ} \mathrm{C}$, maintained at $200{ }^{\circ} \mathrm{C}$ for $5 \mathrm{~min}$, cooled to $-30^{\circ} \mathrm{C}$ and finally reheated to $200{ }^{\circ} \mathrm{C}$. Temperatures were adjusted at a rate of $10^{\circ} \mathrm{C} \cdot \mathrm{min}^{-1}$. The crystallization temperature $\left(\mathrm{T}_{\mathrm{c}}\right)$ determined from cooling ramp, melting temperature $\left(\mathrm{T}_{\mathrm{m}}\right)$ and melting enthalpy $(\triangle \mathrm{Hm})$ obtained from first heating ramp, were determined from the DSC curves using the Universal Analysis 2000 software by TA Instruments. The crystallinity degree $\left(X_{C}\right)$ of neat and nanocomposite polymers was determined by the equation:

$$
\mathrm{X}_{\mathrm{C}}(\%)=\frac{\Delta \mathrm{H}_{\mathrm{m}}}{\Delta \mathrm{H}_{\mathrm{m}}^{0}} \times \frac{100}{\mathrm{w}}
$$

where: $\Delta \mathrm{H}_{\mathrm{m}}$ is the melting enthalpy of the polymer matrix, $\mathrm{w}$ is the polymer weight fraction in the sample and $\Delta \mathrm{H}_{\mathrm{m}}^{0}$ is the theoretical melting enthalpy of the polymer assumed to be $100 \%$ crystalline, $\Delta \mathrm{H}_{\mathrm{m}}^{0}=146.0 \mathrm{~J} \cdot \mathrm{g}^{-1}$ for PHBV [18] and $\Delta \mathrm{H}_{\mathrm{m}}^{0}=116.9 \mathrm{~J} \cdot \mathrm{g}^{-1}$ for PBSA [19].

\subsection{Overall Migration Testing}

Overall migration (OM) level was gravimetrically determined after the total immersion of samples in the selected food simulants based on the standardized testing conditions set out for long term storage $\left(40^{\circ} \mathrm{C}\right.$ for 10 days) in EU Regulation 10/2011. The samples were cut in disks of $2.5 \mathrm{~cm}$ diameter and kept in a vacuum oven at $60^{\circ} \mathrm{C}$ for 2 days. The polymer disks with the total area of $60 \mathrm{~cm}^{2}$ were weighed using a microbalance ( $0.1 \mathrm{mg}$ resolution) prior to the migration test, and then placed in a $100 \mathrm{~mL}$ migration cell (DURAN ${ }^{\circledR}$ screw thread tubes) with rod and glass spacers and PTFE-sealed caps. Before filling, the migration cells were blown out with nitrogen to prevent any dust contamination. The bottles were then filled with the respective simulants, sealed and stored in a $40{ }^{\circ} \mathrm{C}$ oven. Each sample/simulant combination was prepared in triplicate. After 10 days of contact, the bottles were ultrasonicated for $2 \mathrm{~min}$ to detach any potential particles from the sample surface. The samples were 
then removed and placed in a vacuum oven at $60^{\circ} \mathrm{C}$ for 3 days. After evaporation of the simulant, the specimens were weighed again, and overall migration was calculated in milligrams per square decimeter of the surface area of sample.

\subsection{Specific Migration Testing}

\subsubsection{Spiking of Films and Desorption Tests}

The films were spiked by the array of additives by impregnation at $40{ }^{\circ} \mathrm{C}$ over $7 \mathrm{~d}$ under rotary agitation with a theoretical additive amount of $500 \mathrm{mg} \cdot \mathrm{kg}^{-1}$ for each of them. After a quick ethanol rinsing to eliminate surface additives, between 0.5 and $1 \mathrm{~g}$ of contaminated materials were immersed in $100 \mathrm{~mL}$ of food simulants in a surface/volume ratio of $6 \mathrm{dm}^{2} \cdot \mathrm{L}^{-1}$ at $40{ }^{\circ} \mathrm{C}$ for 10 days under magnetic agitation. At the end of the contact time, an aliquot of food simulant was collected and analyzed by GC-FID using an Agilent 7890A GC equipped with a 7693A Automatic Sampler and a flame ionization detector. The components were separated using a HP-5 (5\%-phenyl)methylpolysiloxane capillary column of $32 \mathrm{~mm}$ ID and $30 \mathrm{~m}$ length. The thermal protocol used for the oven was as follows: an initial temperature of $40{ }^{\circ} \mathrm{C}$ maintained for $5 \mathrm{~min}$, heating at a rate of $6{ }^{\circ} \mathrm{C} \cdot \mathrm{min}^{-1}$ to $270{ }^{\circ} \mathrm{C}$ and maintenance for $15 \mathrm{~min}$. The injector temperature was $250^{\circ} \mathrm{C}$. An external calibration was made in each food simulant in the range of 1.25 to $40 \mathrm{mg} . \mathrm{L}^{-1}$.

\subsubsection{Relative Migration}

The relative migration represents the percentage of an additive that migrates from polymer to food simulant. It was calculated by dividing the mass of additive recovered in the food simulant after 10 days of contact by the initial mass of additive in the film sample measured after two consecutive extractions in $20 \mathrm{~mL}$ of $\mathrm{n}$-hexane.

\subsubsection{Statistical Treatment}

Variance analyses were performed using XLSTAT software. Effects of the addition of nanoclays and the UV treatment ( $n=42$ for each material-food simulant couple) were studied by variance analysis regardless of each other and the nature of the additive.

\subsubsection{Estimation of the $D_{a p p}$}

The $D_{\text {app }}$ values of additives from the polymer films into food simulants were calculated from experimental desorption kinetic curves using Crank's mathematical model derived from Fick's second law [20]:

$$
\frac{M_{t}}{M_{\infty}}=1-\sum_{n=0}^{\infty} \frac{2 \alpha(1+\alpha)}{1+\alpha+\alpha^{2} q_{n}^{2}} \exp \left\{-\frac{D_{a p p} q_{n}^{2} t}{L^{2}}\right\}
$$

with

$$
\alpha=\frac{1}{K_{P, F}} \frac{V_{F}}{V_{P}}
$$

where $M_{t}$ is the total amount of additives in food simulant at time $t$ and $M_{\infty}$ is the total amount of additives in the food simulant at the steady state. $V_{P}$ is the polymer volume and $V_{F}$ is the food simulant volume. $\left(q_{n}\right)_{n}$ is the positive root of the equation tan $q=-\alpha q$, and $K_{P, F}$ is the partition coefficient of the additive in the polymer/food simulant system.

The numerical simulations were carried out using Matlab ${ }^{\circledR}$ software and its lsqnonlin function to estimate the $D_{a p p}$. 


\section{Results and Discussion}

\subsection{Semi-Crystalline Structure of Materials}

The crystallization behavior was determined from a heat-cool-heat cycle using DSC for neat, nanocomposite and aged materials. The crystallization temperature $\left(T_{c}\right)$, melting temperature $\left(T_{m}\right)$ and the degree of crystallinity $\left(X_{C}\right)$ are gathered in Table 1.

Table 1. Crystallization temperature $\left(T_{c}\right)$, melting temperatures $\left(T_{m}\right)$ and crystallinity degree $\left(X_{C}\right)$ of neat and nanocomposite PBSA and PHBV and the corresponding aged materials.

\begin{tabular}{llccc}
\hline \multirow{2}{*}{ PBSA } & & $\mathbf{T}_{\mathbf{c}}\left({ }^{\circ} \mathbf{C}\right)$ & $\mathbf{T}_{\mathbf{m}}\left({ }^{\circ} \mathbf{C}\right)$ & $\mathbf{X}_{\mathbf{C}}(\mathbf{\%})$ \\
\hline \multirow{2}{*}{ PBSA NCP } & Control & $42.3 \pm 0.1$ & $86.7 \pm 0.4$ & $45.6 \pm 0.6$ \\
& UV treated & $39.8 \pm 3.7$ & $88.4 \pm 1.8$ & $53.7 \pm 1.9$ \\
\hline \multirow{2}{*}{ PHBV } & Control & $44.0 \pm 0.3$ & $87.2 \pm 0.6$ & $45.8 \pm 0.5$ \\
& UV treated & $33.4 \pm 0.1$ & $86.6 \pm 1.4$ & $53.4 \pm 2.3$ \\
\hline \multirow{2}{*}{ PHBV NCP } & Control & $122.1 \pm 0.1$ & $169.1 \pm 0.7$ & $54.1 \pm 1.2$ \\
& UV treated & $117.8 \pm 0.1$ & $166.1 \pm 0.4$ & $55.5 \pm 0.8$ \\
\hline
\end{tabular}

The results indicate that the aging significantly affected the thermal properties of the materials with roughly similar effects on both polyesters. In the case of PBSA materials, the $\mathrm{T}_{\mathrm{m}}$ values did not appear significantly modified after accelerated aging. In contrast, the crystalline transition was significantly impacted with a decrease and the $X_{C}$ increasing significantly. Concerning the PHBV materials, the $T_{m}$ and $T_{C}$ decreased significantly after aging. The $X_{C}$ tended to increase but to a lesser extent compared to PBSA materials. The effect of irradiation on PBSA [21,22] and PHBV [23-25] has already been studied and similar results were obtained. The decrease of the $T_{C}$, observed with both materials, was attributed to chain scission mechanism leading to the formation of shorter polymer chains. This reduction in chain size should result in an increase in mobility (and therefore freedom of movement) that allows structural rearrangement of polymers in a crystalline state at a lower temperature compared to long polymer chains $[23,26]$. Regarding the $\mathrm{T}_{\mathrm{m}}$, for PHBV, a decrease was observed and double melting peaks appeared, which indicate changes in the crystal structures of polymers. The decrease of $T_{m}$ may also be attributed to the decrease in molar mass of polymer chains, this hypothesis being supported in several studies by the determination of the number average molecular weight $\left(\overline{\mathrm{M}}_{n}\right)$ by size-exclusion chromatography (SEC) [25,27-29]. The increase of the degree of crystallinity observed was as initiated by the scission of polymer chains, due to irradiation, particularly in amorphous regions, which are more accessible for degradation. The higher mobility of these shorter chains allows them to recrystallize easily. This phenomenon was more marked in the case of PBSA, whose degree of crystallinity was initially lower, and consequently, amorphous regions were more important.

Finally, it is important to point out that the increase of the degree of crystallinity was visually confirmed since the materials became very brittle compared to unaged control materials (Figure 1). The addition of nanoclays does not seem to have any effect on the impact of accelerated aging, either on crystallization and melting temperatures or on the degree of crystallinity. From a mechanical point of view, they seem to play a reinforcing role by slightly preserving the ductility of the materials, this effect being more noticeable on the PBSA. 

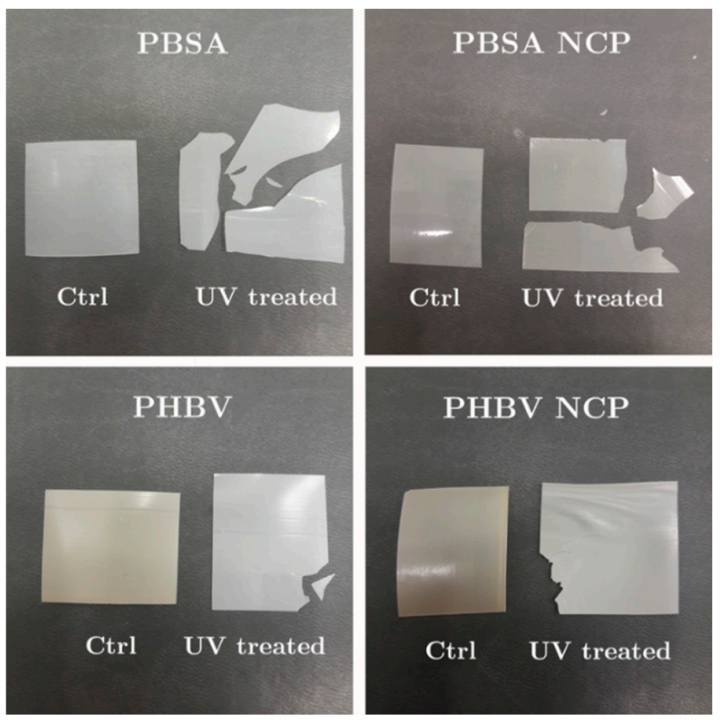

Figure 1. Pictures of the materials before and after accelerated aging.

\subsection{Overall Migration Measure}

According to Article 3 of Regulation (EC) 1935/2004 [30], the release of substances from food contact materials should not bring about unacceptable changes in the composition of the food. That is why the overall migration limit, which represents the maximum permitted amount of non-volatile substances released from a material into foods or food simulants, was set to $10 \mathrm{mg}$ of substances per 1 $\mathrm{dm}^{2}$ of surface area of plastic material.

The results of the overall migration measurements of PBSA, PBSA NCP, PHBV and PHBV NCP, and the corresponding aged materials, are reported in Figures 2 and 3.

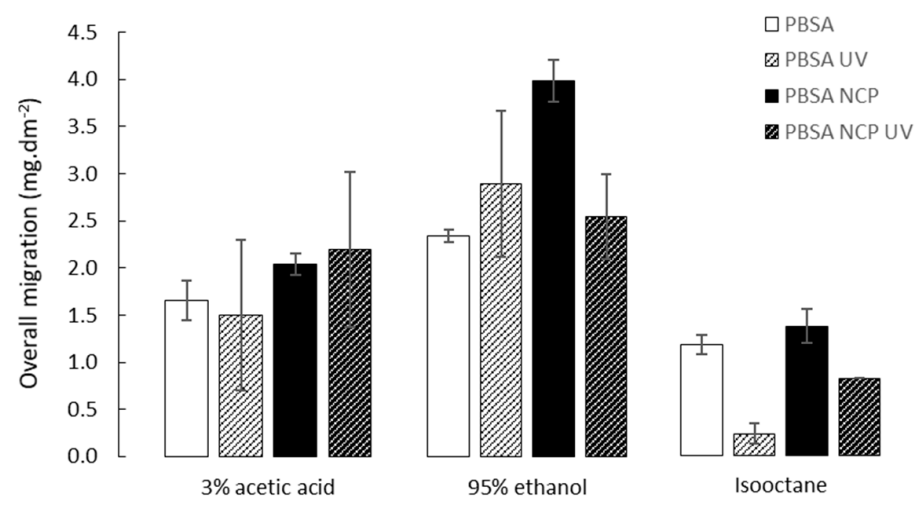

Figure 2. Overall migration of PBSA, PBSA NCP and the corresponding aged materials to the selected food simulants ( $3 \%$ acetic acid, $95 \%$ ethanol and isooctane).

All the studied materials showed an overall migration value lower than the authorized threshold of $10 \mathrm{mg} \cdot \mathrm{dm}^{-2}$. Based on that criterion, these materials meet the level of requirement that determines their allowance for food contact with acidic and fatty foods. Nevertheless, different behaviors were observed depending on the polymer type and the food simulant in contact and are in agreement with the results of previous works on these materials [31]. The contact with $95 \%$ ethanol was already identified as the "worst case" condition most affecting the inertial properties of bio-polyester. The impact of the accelerated aging, when it was significant, decreased the overall migration whether nanoclays were added or not. This can be explained to a large extent by the increase in the degree of crystallinity induced by the treatment evidenced in Section 3.1. Considering that the migration values measured in this experiment correspond mainly to the release of polymer fragments soluble in the various solvents 
used as food simulants, the expansion of the crystalline phase can be considered as an obstacle to the migration process. It should be noted that the impact of UV treatment is particularly significant on the PHBV in contact with ethanol, this result suggesting a combined effect of UV irradiation and ethanol sorption known to induce crystallization of this polymer.

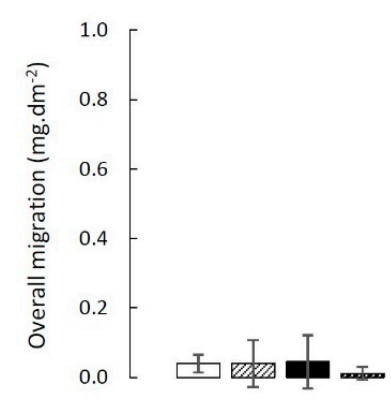

$3 \%$ acetic acid
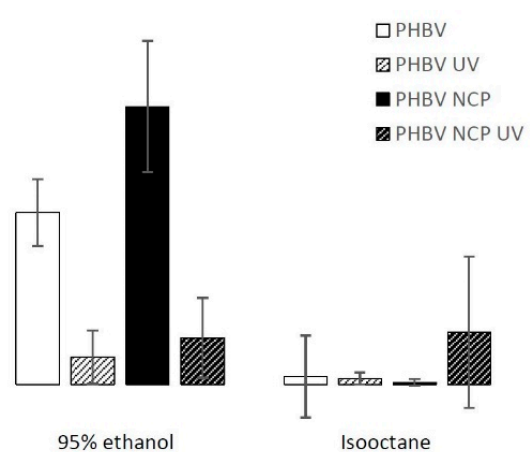

Isooctane

Figure 3. Overall migration of PHBV, PHBV NCP and the corresponding aged materials to the selected food simulants ( $3 \%$ acetic acid, $95 \%$ ethanol and isooctane).

The impact of the addition of nanoclays appears to be variable and only slightly significant in most cases. Overall, the addition of such nanofillers tends to increase the overall migration rate when no UV treatment is applied to materials, no significant behavior being observed after aging of the materials. However, it is established that nanoclays have a nucleating effect which tends to increase the crystallization rate of semi-crystalline thermoplastic polymers [32,33], and should therefore decrease the migration.

\subsection{Relative Migration Measurement}

The results of the relative migration of additives after 10 days of contact with food simulants are gathered in Figures 4 and 5 for PHBV, PHBV NCP (Figure 4), PBSA and PBSA NCP (Figure 5), and the corresponding UV treated materials.

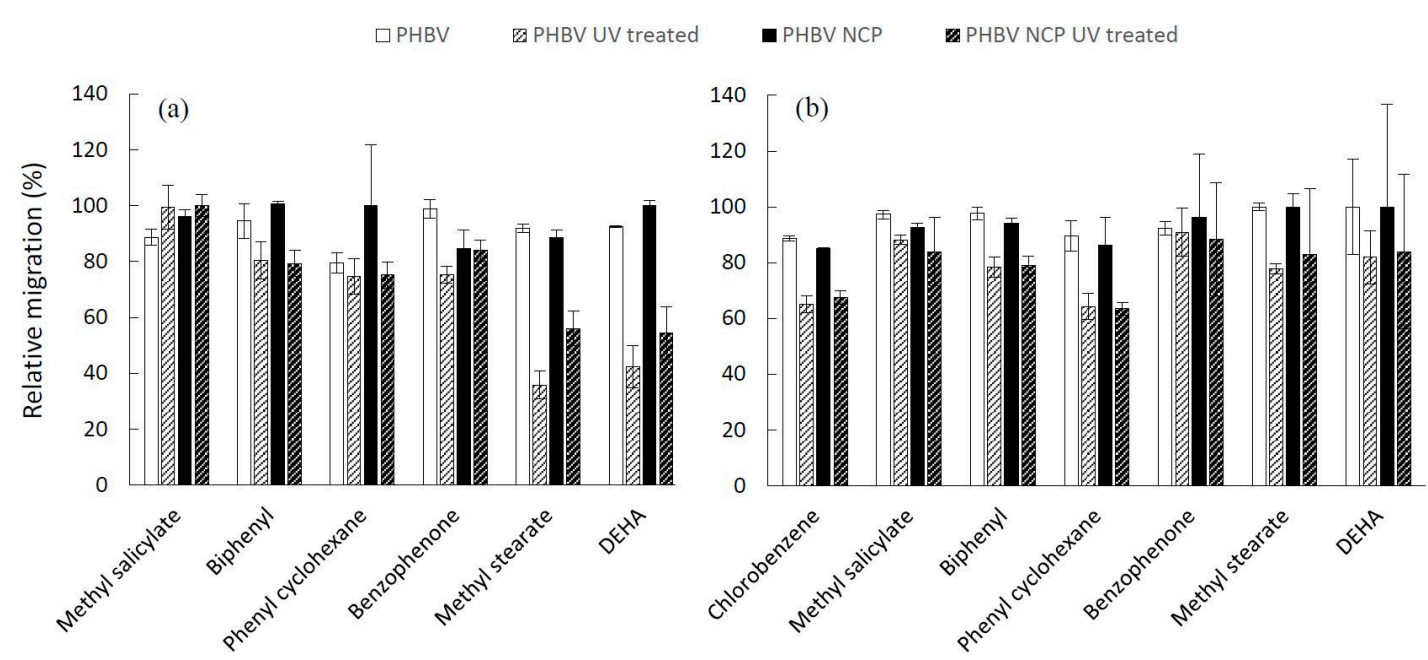

Figure 4. Comparison of the relative migrations of additives from PHBV, PHBV NCP and the corresponding aged materials to $95 \%$ ethanol (a) and isooctane (b) after 10 days of contact.

The relative migration values are generally high, reaching $100 \%$ in several cases, which reflects a high level of contamination in the case of contact with fatty foods. The values are substantially similar in both types of solvents and very close to the values measured with the same target molecules from synthetic materials such as polyolefins [10]. Overall, the level of migration obviously depends on the 
nature of the substance, but each compound behaves differently depending on the type of material and the food simulant in contact. The sorption of such polar simulants is known to induce the swelling of the polymeric network, and consequently, modify the transport properties of the material. In addition, the sorption of non-polar solvent is likely to significantly modify the affinity of the compound for the packaging film, and thus impact its migration level.

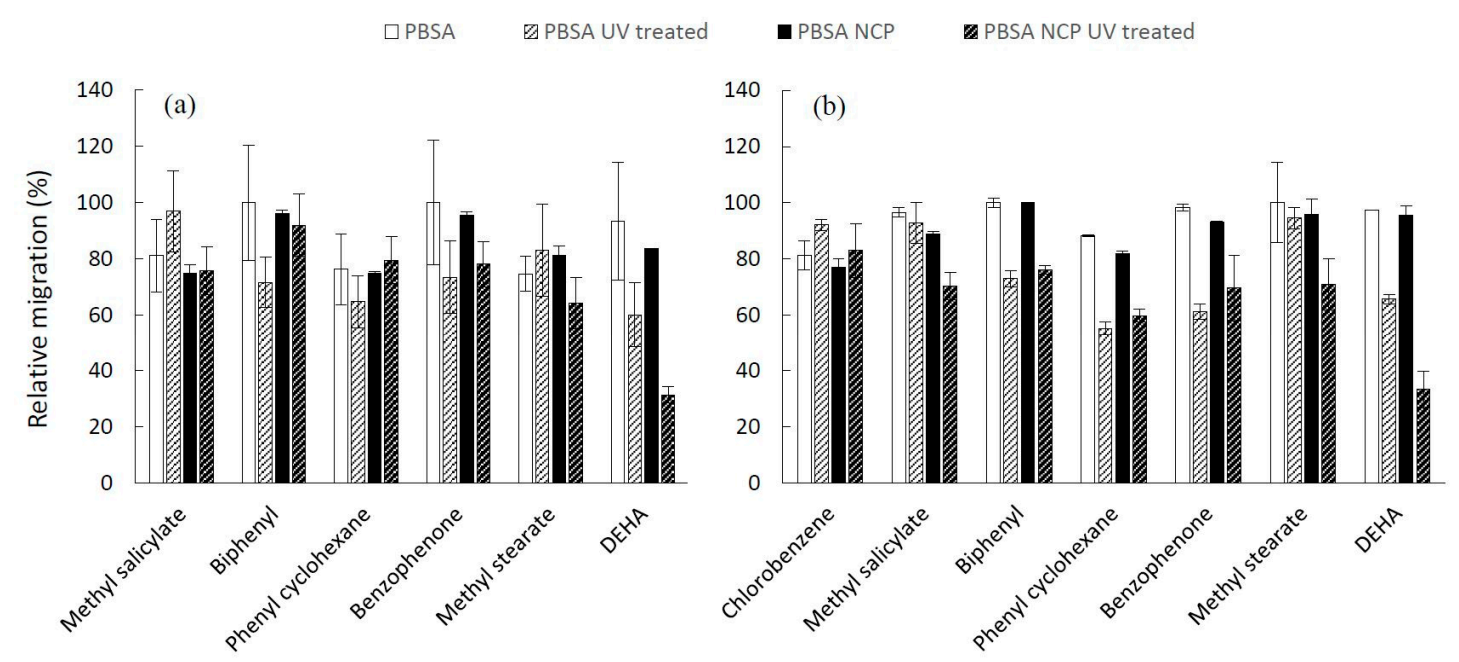

Figure 5. Comparison of the relative migrations of additives from PBSA, PBSA NCP and the corresponding aged materials to $95 \%$ ethanol (a) and isooctane (b) after 10 days of contact.

The impact of the presence of nanoclays is difficult to extract and does not follow any particular pattern. The analysis of variance reveals a variable influence of nanofillers depending on the nature of the material and the simulant. In the particular case of PBSA, the addition of a nanoclay has no significant impact when the packaging is in contact with ethanol, whereas it induces a decrease in the level of migration $(p=0.027)$ as a result of contact with isooctane. On the other hand, the presence of nanoclays does not seem to produce any significant effect on the inertial properties of the PHBV, regardless of the simulant placed in contact. The dispersion of nanoclays is, however, known to impact both the diffusivity of low molar mass molecules by a possible tortuosity effect, and influence the equilibrium state and the partition coefficient by interaction or adsorption effects with the diffusing molecules. For high-barrier polymers in a glassy state at an operating temperature by which the equilibrium is reached slowly, the level of migration measured after only ten days of contact is more specifically related to the diffusion coefficient of the substance. For low-barrier polymers in rubbery states, such as PBSA and PHBV, the measurements carried out after 10 days of contact were performed in an equilibrium state of concentration in both compartments (packaging and food simulant) for each additive. This observation partly justifies the low influence of the presence of nanoclays, whose impact on the diffusion properties of these polymers has already been demonstrated [11].

By looking at each compound independently, the accelerated aging seems to limit the migration of additives. This trend was observed for all substances except in some rare cases (methyl salicylate in PHBV in contact with 95\% ethanol and in PBSA NCP in contact with isooctane). The analysis of variance carried out on all the data regardless of the nature of the additive shows a significant decrease in the migration value $(0.0001<p$-value $<0.016)$ on both types of films in contact with the two tested food simulants. The box plot in the Figure 6 graphically depicts the effect of the UV aging, which is exercised in similar extent on both bio-polyester-based materials. The variance analysis reveals, in addition, a significant interaction $(0.0001 \leq p$-value $\leq 0.014)$ between the UV treatment and the nature of additive, suggesting that the decrease in migration induced by the material aging depends on the nature of the additive. 

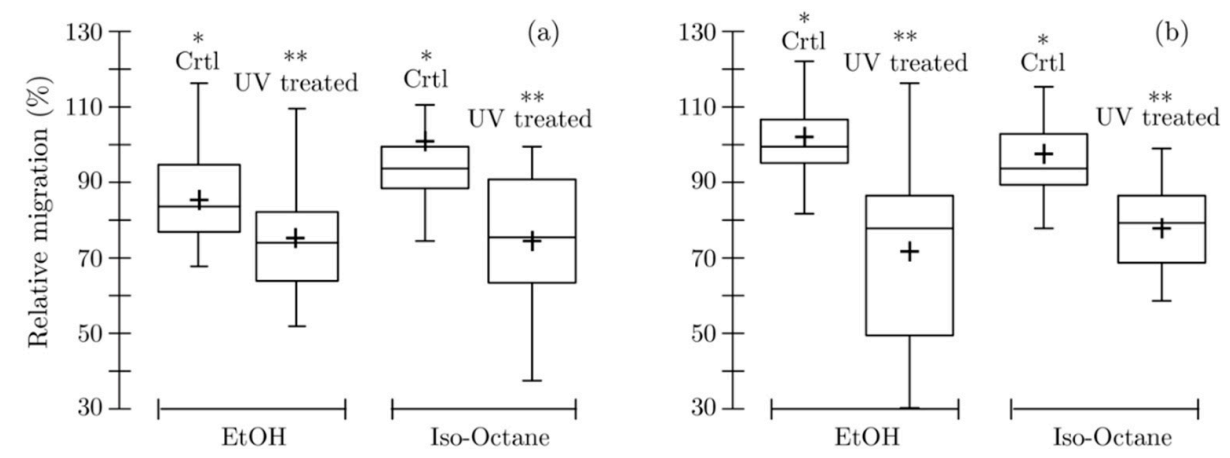

Figure 6. Univariate plot summarizing the distribution of relative migration values as a function of aging treatment applied to neat and nanocomposite PBSA (a) and PHBV (b) materials in contact with $95 \%$ ethanol and isooctane.

This result appears to be in good coherence with the increase of the degree of crystallinity previously observed (Table 1), since as a first approximation and according to the free volume theory describing the diffusion in semi-crystalline polymeric system [34], a higher degree of crystallinity, which leads to a higher percentage of crystalline phase, is positive for the decreasing migration phenomena [35].

On the other hand, it is important to note that no significant interaction $(0.4 \leq p$-value $\leq 0.8)$ was evidenced between the UV treatment and the addition of nanoclays, which implies that the effect of aging treatment is not dependent on the presence of the nanoclays. From a practical point of view, these results provide the basis of the recommendations related to the assessment of migration from biodegradable materials. While the migration test conditions defined for plastic materials may seem inappropriate for biodegradable and bio-composite materials, this study demonstrates that the food simulants and contact conditions established under Regulation 10/2011 are potentially applicable to these particular materials.

\subsection{Dapp Values of Additives in Neat and Nanocomposite Aged Materials}

The estimation of the $\mathrm{D}_{\text {app }}$ of each additive from the biopolymer into fatty food simulants is reported in Figure 7 (PHBV) and Figure 8 (PBSA), showing the respective impacts of: polymer type, food simulant, nanoparticle incorporation and accelerated aging.

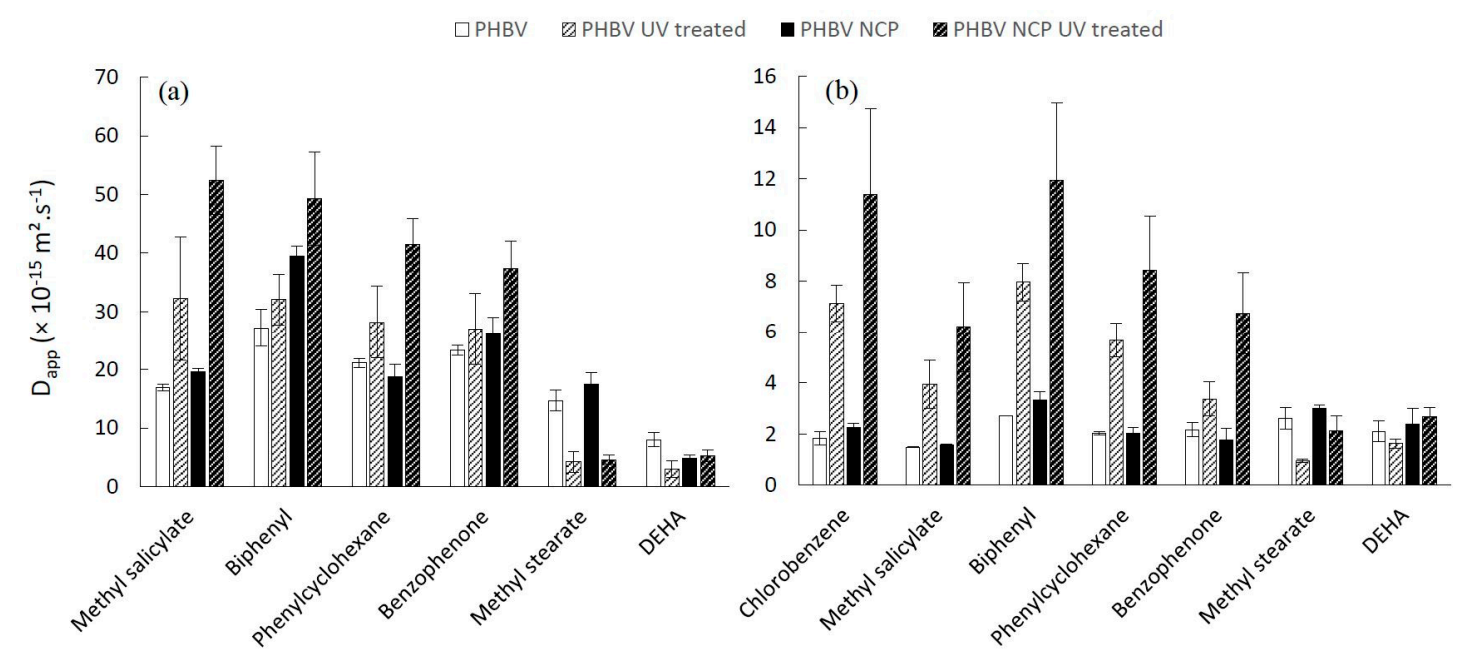

Figure 7. Comparison of $\mathrm{D}_{\text {app }}$ values of additives in PHBV, PHBV NCP and the corresponding aged materials in contact with $95 \%$ ethanol (a) and isooctane (b). 

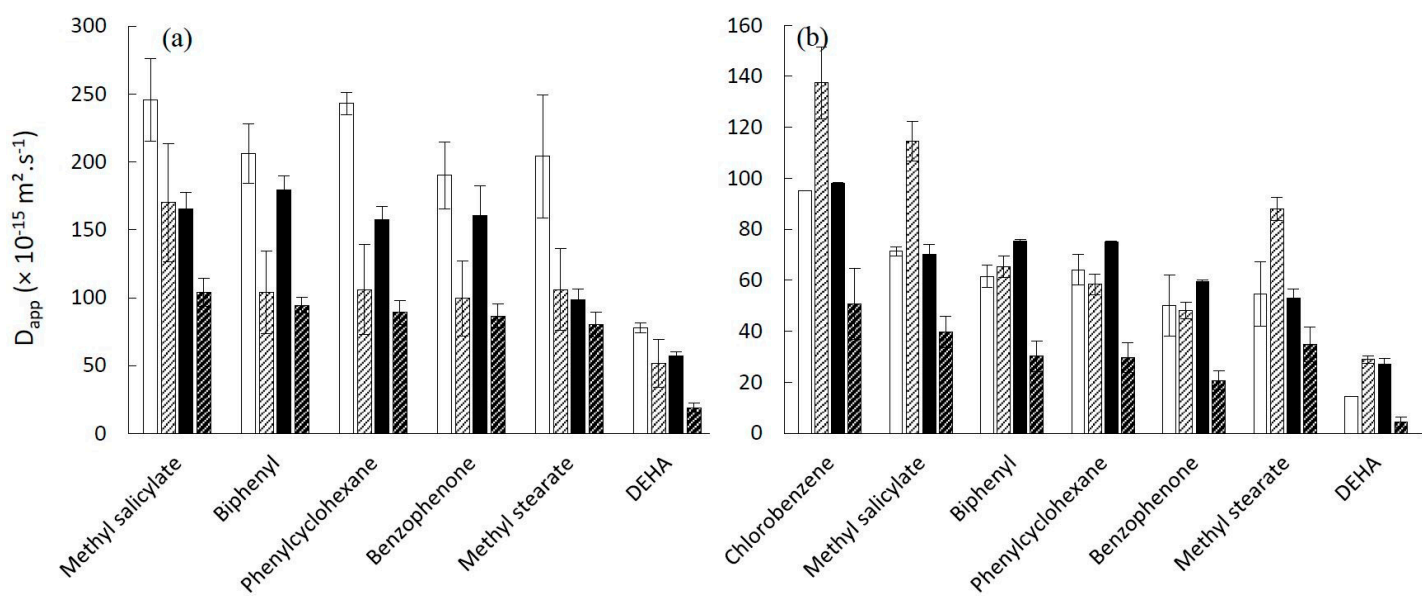

Figure 8. Comparison of $\mathrm{D}_{\text {app }}$ values of additives in PBSA, PBSA NCP and the corresponding aged materials in contact with $95 \%$ ethanol (a) and isooctane (b).

The differing scales of the histograms highlight the impacts of the polymer type and the food simulant, these observations having already been established and commented on a previous study performed on untreated material [11]. The accelerated aging does not impact this range of scales; the apparent diffusion coefficient ranking is as follows: PBSA/95\% ethanol > PBSA/isooctane $>$ PHBV/95\% ethanol $>$ PHBV/isooctane. However, contrasted results due to the treatment of materials were obtained depending on polymer type and food simulant in contact.

The effect of the presence of nanoclays in the PHBV is very significant $(n<0.0001)$, leading to a dramatic increase in the diffusivity values of almost all additives, whatever the food simulant in contact. However, this effect does not apply to the particular case of higher molar mass compounds for which the influence of nanofillers is more difficult to identify. This increase in the diffusivity of low molar mass molecules may appear in opposition to an expected tortuosity effect due to the presence of nanofillers and an increase in crystallinity induced by UV treatment. The most tangible hypothesis to explain this behavior is the macroscopic damage of the films induced by the aging process. It is indeed likely that the accelerated aging of materials will produce microcracks, generating diffusion channels, particularly for the most volatile compounds. This effect should be enhanced by the presence of inorganic nanoclays whose differences in mechanical strength with the polymeric matrix could generate channels at the non-cohesive interface between the two phases.

The effect of UV treatment is noticeably different for PBSA-based materials. For aged PBSA materials in contact with $95 \%$ ethanol (Figure 8a), a significant decrease of the $\mathrm{D}_{\text {app }}$ was observed $(n<0.0001)$ for each additive compared to each unaged one. These results are in accordance with those of the degree of crystallinity reported in Section 3.1. This decrease was additionally observed for PBSA NCP, suggesting an apparent tortuosity effect associated with a nucleating effect of nanoclays. Surprisingly, the UV treatment induces, on the contrary, an increase in the diffusion of the compounds in the neat PBSA when it is placed in contact with isooctane, this behavior being difficult to explain at the scale of observation of this study.

Regarding the influence of nanoclays on aged materials, a conventional impact was observed for PBSA and PBSA NCP; that is, nanoclays decrease the $\mathrm{D}_{\mathrm{app}}$, while a non-conventional impact was evidenced in the case of PHBV and PHBV NCP.

These results suggest that the degree of crystallinity is not sufficient to explain the transfer properties of a material, since the measure of the crystallinity degree measured on all of the samples does not evidence any impact of the incorporation of nanoclays. It is therefore likely that the combined effects of nanoclay and UV treatment induce structural reorganizations of polymer chains which need 
to be analyzed at a finer scale, with the diffusivity of additives in a semi-crystalline polymer, such as PBSA and PHBV, being potentially affected by:

- The ratio between the mobile and rigid amorphous fractions of a semi-crystalline polymer (commonly named MAF and RAF, respectively). In the MAF, when the polymers are in the rubbery state, the polymer chains are free to move. Consequently, the diffusivity is increased0, whereas the polymer chains' mobility is hindered at the neighborhood of crystalline phase and nanoclays in RAF [36].

- The size of the crystals in the crystalline phase. Larger crystals lead to an increase of diffusion pathways, and therefore a decrease of the diffusivity. Charlon et al. evidenced this hypothesis by studying the barrier properties of PBS and PBSA [36].

It is assumed that UV radiation induces very local changes of state that are not detected by a global measurement of the degree of crystallinity. Since the presence of nanoclays can also modify the assembly of the polymer network, a refined observation of materials on a nanoscale seems necessary to characterize the diffusion mechanisms that take place in these structures.

\section{Conclusions}

The use of nanocomposite materials as food packaging raises new questions in terms of consumer safety. This is even more true with the use of biodegradable polymers for which the structures are, by definition, instable over time. It was not a priori advisable to reproduce the conditions of migration testing developed and applied to plastic materials in the same way. This study was performed to verify the food contact suitability of bio-nanocomposite materials after critical accelerated aging following the recommendations of Regulation (EU) 10/2011 defined for non-degradable polymers. The structures of materials proved to be affected by the treatment, but the consequences on the properties of transfer and inertia of materials are not deleterious regarding the safety issues of such materials. The increase of the degree of crystallinity induced by the UV irradiation leads to a decrease of the overall migration. Except for a few rare cases, a lower specific migration of additives was also observed after aging, regardless of the additive, the nature of the polymer and the food simulant in contact. This decrease in migration is not related to a decrease in the diffusivity of additives, which is in most cases, subject to an increase despite the increase in the crystallinity of the material measured and the presence of impermeable nanoclays likely to exert a barrier effect. As consequence, these results do not call into question the food contact suitability of these materials, even as they age. From a practical point of view, this study demonstrates that the recommendations provided by Regulation (EU) 10/2011 are potentially applicable to the particular case of biodegradable materials, the critical point remaining, rather, at the level of the mechanical properties of the materials after aging. They become brittle and difficult to use for packaging applications.

Author Contributions: The research presented in this paper is a collaborative work among all authors. Conceptualization, A.L. and S.P.; methodology, A.L. and S.P.; software, A.L. and S.G.; writing-original draft preparation, A.L. and S.P.; writing-review and editing, S.P. and A.L.; supervision, N.G. and S.P.; funding acquisition, N.G. All authors have read and agreed to the published version of the manuscript.

Funding: The project leading to this publication has received funding from Excellence Initiative of Aix-Marseille University-A*MIDEX, a French "Investissements d'Avenir" program, through its associated with the Labex SERENADE project.

Acknowledgments: The authors would like to gratefully acknowledge Sylvie Motellier, Arnaud Guiot and Sébastien Artous from University Grenoble Alpes, CEA, Lab Nanocharacterization and Nanosafety (Grenoble, France) for the management of the accelerated aging tests.

Conflicts of Interest: The authors declare no conflict of interest.

\section{References}

1. Xia, Y.; Rubino, M.; Auras, R. Release of nanoclay and surfactant from polymer-clay nanocomposites into a food simulant. Environ. Sci. Technol. 2014, 48, 13617-13624. [CrossRef] [PubMed] 
2. Echegoyen, Y.; Rodríguez, S.; Nerín, C. Nanoclay migration from food packaging materials. Food Addit. Contam. Part A 2016, 33, 530-539. [CrossRef] [PubMed]

3. Duncan, T.V.; Pillai, K. Release of engineered nanomaterials from polymer nanocomposites: Diffusion, dissolution, and desorption. Appl. Mater. Interfaces 2015, 7, 2-19. [CrossRef] [PubMed]

4. Handy, R.D.; Shaw, B.J. Toxic effects of nanoparticles and nanomaterials: Implications for public health, risk assessment and the public perception of nanotechnology. Health Risk Soc. 2007, 9, 125-144. [CrossRef]

5. Šimon, P.; Chaudhry, Q.; Bakoš, D. Migration of engineered nanoparticles from polymer packaging to food-A physicochemical view. J. Food Nutr. Res. 2008, 47, 105-113.

6. Bott, J.; Störmer, A.; Franz, R. A model study into the migration potential of nanoparticles from plastics nanocomposites for food contact. Food Packag. Shelf Life 2014, 2, 73-80. [CrossRef]

7. Bott, J.; Franz, R. Investigations into the potential abrasive release of nanomaterials due to material stress conditions-Part B: Silver, titanium nitride, and laponite nanoparticles in plastic composites. Appl. Sci. 2019, 9, 221. [CrossRef]

8. Farhoodi, M.; Mohammadifar, M.A.; Mousavi, M.; Sotudeh-Gharebagh, R.; Emam-Djomeh, Z. Migration kinetics of ethylene glycol monomer from PET bottles into acidic food simulant: Effects of nanoparticle presence and matrix morphology. J. Food Process Eng. 2017, 40, e12383. [CrossRef]

9. de Abreu, D.A.P.; Cruz, J.M.; Angulo, I.; Losada, P.P. Mass transport studies of different additives in polyamide and exfoliated nanocomposite polyamide films for food industry. Packag. Technol. Sci. 2010, 23, 59-68. [CrossRef]

10. Nasiri, A.; Peyron, S.; Gastaldi, E.; Gontard, N. Effect of nanoclay on the transfer properties of immanent additives in food packages. J. Mater. Sci. 2016, 51, 9732-9748. [CrossRef]

11. Lajarrige, A.; Gontard, N.; Gaucel, S.; Samson, M.-F.; Peyron, S. The mixed impact of nanoclays on the apparent diffusion coefficient of additives in biodegradable polymers in contact with food. Appl. Clay Sci. 2019, 180, 1-9. [CrossRef]

12. Singh, B.; Sharma, N. Mechanistic implications of plastic degradation. Polym. Degrad. Stab. 2008, 93, 561-584. [CrossRef]

13. Rydz, J.; Musioł, M.; Janeczek, H. Thermal analysis in the study of polymer (bio)-degradation. In Reactions and Mechanisms in Thermal Analysis of Advanced Materials; John Wiley \& Sons, Inc.: Hoboken, NJ, USA, 2015; pp. 103-126.

14. Ramis, X.; Cadenato, A.; Salla, J.M.; Morancho, J.M.; Vallés, A.; Contat, L.; Ribes, A. Thermal degradation of polypropylene/starch-based materials with enhanced biodegradability. Polym. Degrad. Stab. 2004, 86, 483-491. [CrossRef]

15. Speight, J.G. Chapter 14-Monomers, Polymers, and Plastics. In Handbook of Industrial Hydrocarbon Processes; Elsevier: Amsterdam, The Netherlands, 2011; pp. 499-537.

16. European Food Safety Authority. Scientific Opinion on the Criteria to Be Used for Safety Evaluation of a Mechanical Recycling Process to Produce Recycled PET Intended to Be Used for Manufacture of Materials and Articles in Contact with Food; European Food Safety Authority: Parma, Italy, 2011.

17. Food and Drug Administration. Guidance for Industry: Use of Recycled Plastics in Food Packaging (Chemistry Considerations); Food and Drug Administration: Silver Spring, MD, USA, 2006.

18. Barham, P.J.; Keller, A.; Otun, E.L. Crystallization and morphology of a bacterial thermoplastic: Poly-3-hydroxybutyrate. J. Mater. Sci. 1984, 19, 2781-2794. [CrossRef]

19. Nikolic, M.S.; Djonlagic, J. Synthesis and characterization of biodegradable poly(butylene succinate-co-butylene adipate)s. Polym. Degrad. Stab. 2001, 74, 263-270. [CrossRef]

20. Crank, J. The Mathematics of Diffusion; Oxford University Press: Oxford, UK, 1975.

21. Zhang, Y.; Xu, J.; Guo, B. Photodegradation behavior of poly(butylene succinate-co-butylene adipate)/ZnO nanocomposites. Colloids Surfaces A Physicochem. Eng. Asp. 2016, 489, 173-181. [CrossRef]

22. Cai, L.; Qi, Z.; Xu, J.; Guo, B.; Huang, Z. Study on the photodegradation stability of poly(butylene succinate-co-butylene adipate)/TiO2 nanocomposites. J. Chem. 2019, 2019, 1-9. [CrossRef]

23. Oliveira, L.M.; Araujo, E.S.; Guedes, S.M.L. Gamma irradiation effects on poly(hydroxybutyrate). Polym. Degrad. Stab. 2006, 91, 2157-2162. [CrossRef]

24. Rosario, F.; Corradini, E.; Casarin, S.A.; Agnelli, J.A.M. Effect of gamma radiation on the properties of poly(3-Hydroxybutyrate-co-3-Hydroxyvalerate)/Poly(e-Caprolactone) blends. J. Polym. Environ. 2013, 21, 789-794. [CrossRef] 
25. Iggui, K.; Kaci, M.; le Moigne, N.; Bergeret, A. The effects of accelerated photooxidation on molecular weight and thermal and mechanical properties of PHBV/Cloisite 30B bionanocomposites. J. Renew. Mater. 2018, 6, 288-298. [CrossRef]

26. Hermida, E.B.; Mega, V.I.; Yashchuk, O.; Fernandez, V.; Eisenberg, P.; Miyazaki, S.S. Gamma irradiation effects on mechanical and thermal properties and biodegradation of poly(3-hydroxybutyrate) based films. Macromol. Symp. 2008, 263, 102-113. [CrossRef]

27. Mitomo, H.; Watanabe, Y.; Ishigaki, I.; Saito, T. Radiation-induced degradation of poly(3-hydroxybutyrate) and the copolymer poly(3- hydroxybutyrate-co-3-hydroxyvalerate). Polym. Degrad. Stab. 1994, 45, 11-17. [CrossRef]

28. Zembouai, I.; Kaci, M.; Bruzaud, S.; Dumazert, L.; Bourmaud, A.; Mahlous, M.; Lopez-Cuesta, J.M.; Grohens, Y. Gamma irradiation effects on morphology and properties of PHBV/PLA blends in presence of compatibilizer and Cloisite 30B. Polym. Test. 2016, 49, 29-37. [CrossRef]

29. Zembouai, I.; Kaci, M.; Bruzaud, S. The influence of gamma irradiation on the chemical structure and crystallinity of PHBV/PLA/Cloisite 30B nanocomposites. AIP Conf. Proc. 2019, 2055, 1-5.

30. European Commission. Regulation No 1935/2004 of the European Parliament and of the Council of 27 October 2004 on Materials and Articles Intended to Come into Contact with Food; European Commission: Strasbourg, France, 2004; pp. 4-17.

31. Chea, V.; Angellier-Coussy, H.; Peyron, S.; Kemmer, D.; Gontard, N. Poly(3-hydroxybutyrate-co-3-hydroxyvalerate) films for food packaging: Physical-chemical and structural stability under food contact conditions. J. Appl. Polym. Sci. 2015, 133, 1-8. [CrossRef]

32. Ke, Y.; Long, C.; Qi, Z. Crystallization, properties, and crystal and nanoscale morphology of PET-clay nanocomposites. J. Appl. Polym. Sci. 1999, 71, 1139-1146. [CrossRef]

33. Yuan, Q.; Awate, S.; Misra, R.D.K. Nonisothermal crystallization behavior of melt-intercalated polyethylene-clay nanocomposites. J. Appl. Polym. Sci. 2006, 102, 3809-3818. [CrossRef]

34. Sharma, J.; Tewari, K.; Arya, R.K. Diffusion in polymeric systems-A review on free volume theory. Prog. Org. Coat. 2017, 111, 83-92. [CrossRef]

35. Dardmeh, N.; Khosrowshahi, A.; Almasi, H.; Zandi, M. Study on effect of the polyethylene terephtalate/nanoclay nanocomposite film on the migration of terephtalic acid into the yoghurt drinks simulant. J. Food Process Eng. 2015, 40, e12324. [CrossRef]

36. Charlon, S.; Marais, S.; Dargent, E.; Soulestin, J.; Sclavons, M.; Follain, N. Structure-barrier property relationship of biodegradable poly(butylene succinate) and poly[(butylene succinate)-co-(butylene adipate)] nanocomposites: Influence of the rigid amorphous fraction. Phys. Chem. Chem. Phys. 2015, 17, 29918-29934. [CrossRef]

(C) 2020 by the authors. Licensee MDPI, Basel, Switzerland. This article is an open access article distributed under the terms and conditions of the Creative Commons Attribution (CC BY) license (http://creativecommons.org/licenses/by/4.0/). 